\title{
General Theory of Body Contouring: 2. Modulation of Mechanical Properties of Subcutaneous Fat Tissue
}

\author{
Ilja L. Kruglikov \\ Wellcomet GmbH, Karlsruhe, Germany \\ Email: $\underline{\text { i.kruglikov@wellcomet.de }}$
}

Received 25 February 2014; revised 20 March 2014; accepted 26 March 2014

Copyright (C) 2014 by author and Scientific Research Publishing Inc.

This work is licensed under the Creative Commons Attribution International License (CC BY). http://creativecommons.org/licenses/by/4.0/

(c) (i) Open Access

\begin{abstract}
Subcutaneous white adipose tissue (sWAT) can be described micromechanically as a foam structure. It is shown that according to this model, mechanical stiffness of this tissue is primarily dependent on the average cell size and is almost independent of the dispersion of cell sizes in a local adipocytes' population. Whereas the influence of natural fat renewal process with a rate of $10 \%$ per year must be of minor importance for mechanical properties of sWAT, induced adipocytes' death can substantially reduce local sWAT stiffness. The sWAT which contains two or more different subpopulations of adipocytes of varying sizes with a spatially clustered structure can demonstrate significant inhomogeneity of their mechanical properties when compared with those of sWAT consisting of a single population of adipocytes. It is proposed that this effect may be an important pathophysiological feature of cellulite. Transformation of the cell shape from quasispherical to wrinkled or elliptical forms makes adipocytes more susceptible to thermo-mechanical stress reducing the strain needed to achieve the local plastic deformation. These mechanical features of sWAT are essential for understanding the mechanisms of different non-invasive and minimal invasive body contouring procedures.
\end{abstract}

\section{Keywords}

Subcutaneous Adipose Tissue; Mechanical Properties; Stiffness; Susceptibility to Plastic Deformation; Adipocytes Size Distribution; Body Contouring

\section{Introduction}

Subcutaneous white adipose tissue (sWAT) is an important target for different aesthetic treatment methods, es- 
pecially for those connected with body contouring. These methods include applications of different light sources, ultrasound, mechanical compression and tension, low and high temperatures, radio-frequency currents, etc. The main purpose of these methods is the long-term reduction of sWAT volume or modification of its mechanical properties through the improvement of local stiffness of the tissue (the so-called "tightening" effect). Although these methods include applications of different types of energy and different physical forces, they often claim comparable end results, which clearly demonstrate the existence of one or more non-specific components in sWAT reaction. As discussed in the first article [1], the modulation of sWAT volume cannot be characterized by a single physiological reaction with dispersed reaction times, thus supposing that various dynamic processes with different characteristic reaction times must be involved.

Subcutaneous WAT consists mainly of mature adipocytes, which are almost hexagonal cells filled with triglycerides. Adipocytes from one local fat depot demonstrate a broad cell volume distribution [2] with coefficient of variation normally exceeding $40 \%$. This distribution can physiologically vary temporally and spatially thus shifting its average value into the direction of smaller or bigger adipocytes, which can be mainly connected with processes of lipolysis and lipogenesis in sWAT, respectively. Moreover, this tissue is self-renewable with an average turnover rate of approximately $10 \%$ per year [3] [4]. Since adipocytes have practically no cytoskeletal structures which can support and stabilize their form, these cells mainly react to circumferential changes (e.g. to osmotic pressure) through their size modulation. This produced a widespread opinion that bigger adipocytes are far more unstable than the smaller ones (see e.g. [5]) and will preferentially react to application of mechanical stress with rupturing of their membranes. This effect is normally theoretically reasoned by accumulation of the tension in membranes of adipocytes according to Laplace's law, which is higher in big adipocytes with thin membranes. Since $2 \%$ stretching of the surface of a lipid membrane would be enough for its rupture [6], such selectivity looks to be possible even under quasi-physiological conditions. This led to speculation concerning the various treatment procedures in obesity and body contouring which claimed selective damage and removal of big adipocytes with consequent metabolic and mechanical improvement of sWAT.

This oversimplified picture does not take into account other components of sWAT, which are also essentially evolved into its micromechanical structure. In this context, the extracellular matrix with its intercellular (septae) and pericellular collagen structures around mature adipocytes having different spatial distributions and mechanical properties is especially important [7] [8]. Since triglycerides behave as a linear viscous fluid with elastic shear modulus which is much less than that of adipose tissue [7], contribution of lipids into mechanical stiffness of sWAT can be neglected, even though they occupy the main volume of this tissue.

In this paper, we will consider sWAT as foam with combined closed-cell and open-cell structures similar to the description done in [7]. We will however modify this model including the distribution of cell sizes, inclusions and spatial inhomogeneity of adipocytes. All these features are not only present in a sWAT which is intact, but they can be also modulated thermo-mechanically by local applications of different types of energy to sWAT. The main purpose of this article is to reveal a possible influence of this micromechanical structure onto the results of some non-invasive and minimal invasive body contouring procedures.

\section{Subcutaneous WAT as A Foam Structure}

\subsection{Micromechanical Description}

Micromechanically sWAT can be described as a closed-cell foam, i.e. the material containing the spatially localized pockets (adipocytes) filled with triglycerides and surrounded by rigid structures composed of adipocyte's membrane reinforced by pericellular collagen network [7]. Size of a unit cell in such foam corresponds to the typical size of adipocytes which varies between 50 and $150 \mu \mathrm{m}$. Adipocytes in sWAT can have spatially heterogeneous cell size distribution demonstrating a clustered structure. For example, it is known that the "beige" cells which can be produced in sWAT during transdifferentiation of white adipocytes into the cells with brown phenotype are normally produced in spatially correlated clusters. Whereas the reason for such cell clustering is not clear, its existence points to a spatial correlation in the adipocytes' production and expansion. This structural heterogeneity can sufficiently influence the local mechanical properties of sWAT. Recently such heterogeneity on a microscopic scale reflecting also the mechanical properties of sWAT was observed in [9].

Pericellular network reinforcing the basement membrane of adipocytes consists predominantly of collagen types IV (basal lamina) and VI (microfibrills), which are both non-fibrillar in nature. The presence of collagen VI is especially important for limitation of adipocytes' hypertrophy which can be clearly seen in collagen VI 
knockout models, whereas the degree of this limitation is strongly dependent on collagen VI content [10] [11]. Actually, strong pericellular fibrosis is considered to be a hallmark of metabolically changed hypertrophic adipocytes [12]. This reinforced collagen structure can increase by up to three to seven times with increased BMI, which points to the significant modulation of mechanical properties of sWAT in obesity. Whereas this structure is mainly responsible for mechanical characteristics of sWAT and is supposed to be of constant thickness in [7], it can indeed have very variable characteristics. This hallmark of sWAT was recently demonstrated in human sWAT [9].

Lack of collagen VI leads to a significant reduction in the stiffness of pericellular matrix with consequent decrease of the Young's modulus of sWAT. Although no measurements of this correlation were directly completed in sWAT, this relationship is known from other tissues. For example, whereas the pericellular matrix in articular cartilage of normal mice has the Young's modulus of approximately $300 \mathrm{kPa}$, in the same structure in collagen VI knockout mice, this modulus is reduced to the value less than $100 \mathrm{kPa}$ [13]. Therefore, normal and hypertrophic adipocytes must not only have different topological characteristics, but also different stiffness of their pericellular structures, making the mechanical characteristics of a mixed population of size distributed adipocytes strongly heterogeneous.

Another collagen network in sWAT is located intercellular, has the fibrillar nature and consists mainly of collagen types I and III, producing the thick collagen bundles [12]. Such coarse-mesh structure with typical unit cellular size of several millimetres has no closed boundaries forming the open-cell foam structure [7]. Mechanical properties of this structure are significantly worse, as corresponding properties provided by the network of reinforced basement membranes. This reflects the general difference in mechanical properties between closedand open-cell structures.

\subsection{Mechanical Properties of Foams}

Foams are the special types of cellular solids which are widely used in different technical applications and have special mechanical, thermic and electrical properties. These properties are strongly dependent on the properties of the material constructing the cell walls (analogue properties of reinforced basement membrane in sWAT), topology of the foam (form and type of a unit cell, its size, contact between the cells) and the relative density of the foam, $\tilde{\rho} / \rho_{s}$, where $\tilde{\rho}$ is the effective density of the foam and $\rho_{s}$ is the material density of the walls.

Mechanical properties of foams of different topology were summarized in [14]. For the foams of low relative density containing isotropic cells, the closed-cell structure demonstrates the upper limit of stiffness which is simply

$$
\tilde{E} / E_{s} \cong \frac{1}{3} \tilde{\rho} / \rho_{s}
$$

Here $\tilde{E}$ is the effective Young's modulus of the foam, $E_{s}$ is the Young's modulus of the wall material corresponding in the case of sWAT the modulus of reinforced basement membrane of adipocytes.

Yield strength, $\sigma$, is yet another important characteristic of the foam, which describes the stress at which the material begins to deform plastically, demonstrating a non-reversible shape modulation in response to applied forces. For the closed-cell foam of small relative density, the ratio $\tilde{\sigma} / \sigma_{s}$ can be described by a relationship which is very similar to Equation (1).

Adipocytes in sWAT have almost hexagonal forms. To simplify the analysis, we will however consider them as ideal contacting cubes of the length $l=2 R$, where $R$ is an effective adipocyte's size (analogue cell radius). The difference in this geometry to the hexagonal topology can be described by a constant factor. Furthermore we will consider the cubic foams forming the closed-cell structure with variable cell sizes. Since the visco-elastic properties of triglycerides inside of adipocytes have a minor contribution into the mechanical properties of sWAT, when compared to the contribution of collagen network [7], we will further neglect the lipid content considering sWAT to be the quasi-hollow cubic foam.

Relative density of non-strained foam constructed from cubic cells with characteristic dimensions of $2 R$ surrounded by pericellular matrix of thickness $h$ is

$$
\frac{\tilde{\rho}}{\rho_{\mathrm{s}}}=3\left(\frac{h}{R}\right)
$$


From (1) and (2), the Young's modulus and the yield strength of the non-deformed closed-cell structure of low relative density can be presented in the form

$$
\tilde{E} / E_{s} \cong \frac{h}{R}, \quad \tilde{\sigma} / \sigma_{s} \cong \frac{h}{R}
$$

\section{Parameters Influencing the Quasi-Static Mechanical Properties of sWAT}

Relations (3) describe the mechanical properties of idealized perfect sWAT, which consists of a homogeneous population of adipocytes, while having no imperfections. In a real-life situation there must be significant deviations from this model connected with

- dead cells with ruptured membranes;

- inclusions of high rigidity;

- variations in cell topology through wrinkling or deformation of cell membranes;

- significant variation in cell sizes;

- the spatially inhomogeneous distribution of cells of different sizes with different thicknesses of reinforced basement membrane, etc.

\subsection{Imperfections}

Production of regions of reduced density ("holes") in the tissue causes concentration of strains near their surfaces and can lead to reduction of sWAT stiffness. One important reason for the production of such holes is the appearance of dead adipocytes with ruptured membranes and removed pericellular structures. These cells do not contribute into the total stiffness of the tissue thus reducing its effective value. To assess this reduction we apply the rule of mixtures for composite materials. According to this, the overall mechanical modulus of the composed system can be presented in the form

$$
\tilde{E}=\mu \widetilde{E_{A}}+(1-\mu) \widetilde{E_{D}},
$$

where $\widetilde{E_{A}}$ and $\widetilde{E_{D}}$ are the effective Young's moduli of sWAT constructed from alive and dead adipocytes, respectively, and $\mu$ is the volume fraction of alive adipocytes in a local sWAT structure. Taking $\widetilde{E_{A}}$ to be the effective modulus of the perfect structure given by (3) and $\widetilde{E}_{D}$ equals 0 , which means that the whole pericellular structure around adipocytes was removed and does not contribute into the stiffness of the system, we can conclude that appearance of dead adipocytes will effectively reduce the stiffness of the tissue by the factor, $\mu$.

To assess the value of this reduction, we deduce that the remodelling rate of adipocytes in a normal sWAT to be about $10 \%$ per year [3]. Assuming the dead adipocytes will be removed in approximately 1 - 2 weeks, the amount of these cells in sWAT under physiological conditions can be assessed to be less than $0.5 \%$. This estimation is in agreement with observations that in murine sWAT the ratio of dead adipocytes does not exceed 3\% even in diet-induced obesity [15] [16].

However, in the same murine model [16], initially low adipocyte death rate of $0.1 \%$ in epididymal fat depot at week 1 reached $16 \%$ at week 12 and even $80 \%$ at week 16, and then it reduced back to the level of $16 \%$ at week 20. These death rates correlated significantly with adipocytes' sizes. In this case, the reduction of tissue stiffness through adipocytes' death cannot be neglected anymore. It is remarkable that the pericellular collagen deposition in these experiments strongly correlated with the rate of cell death, being at the highest at week 16 and demonstrating significant reduction by week 20. According to the model described above, such behaviour is needed to compensate the loss of the mechanical stiffness in the fat tissue caused by adipocytes' death through increase of pericellular thickness of surviving cells. Indeed, according to (3) and (4), the presence of $50 \%$ of dead adipocytes in the tissue can be fully mechanically compensated by doubling the thickness of pericellular layer around survived adipocytes.

Therefore, local mechanical stiffness of sWAT can effectively be reduced by short-term induced adipocytes' death, followed by the removal of their pericellular network. Different non-invasive and minimal invasive treatment methods in body contouring claim the main mechanism of their application is just the induced death and removal of adipocytes from sWAT. From the above it can be assumed that following the procedure such an effect (if realized) would at least, in the short-term, decrease the effective stiffness of the tissue, which would generally be the opposite to the expected treatment result. 
The opposite effect can be produced by rigid inclusions. Such inclusions can appear, for example, by the application of osmotic stress. Increased osmotic pressure will lead to the influx of the water into the adipocytes, which consequently increases their volume. Since adipocytes in sWAT are limited in their expansion through surrounding cells, they will be forced to increase their turgor to interrupt the further influx of water. Turgor increase by the same deformation of adipocytes will cause effective increase of their Young's modulus, thus increasing the effective stiffness of sWAT. This seemingly paradoxical behaviour of sWAT was recently observed in [9].

\subsection{Cell Size Distribution}

sWAT does not consist of uniform cells and generally demonstrates the broad cell volume distributions in different depots [2]. From (3), it should be intuitively clear that for the same thickness of reinforced basement membrane around adipocytes the regions with a high local concentration of small cells will demonstrate higher stiffness than the regions containing the bigger cells. At the same time, from the general theory of cellular solids it is known that such dispersion of cell sizes does not significantly influence the mechanical properties of closed-cell foams. Indeed, foams consisting of symmetrical cells with a broad size distribution normally demonstrate the properties which are similar to the materials consisting of idealized periodic uniform cells.

The effective Young's modulus of sWAT taking into account the adipocytes' size distribution can be described by relation (A.4) (see Appendix). It is seen that reduction of the average cell size by the same thickness of reinforced basement structure around the adipocytes, $h$, will increase the Young's modulus and thus the stiffness of sWAT. This effect must be observed by reduction of adipocytes' sizes through induced lipolysis without accompanying cell death, which can be realized, for example, by the application of hyaluronidase to a hypertrophic fat tissue (see [17] [18]). This result is quite the opposite to the prediction made in a previous section, where it was shown that the pure cell death without cell size reduction must cause the decrease of the fat tissue stiffness. This phenomenon clearly indicates that different treatment strategies in body contouring can produce opposite treatment results regarding the stiffness of sWAT.

Another effect predicted by (A.4) is the increased effective stiffness of sWAT which consists of adipocytes with a broad distribution of cell sizes. Indeed, for two sWAT depots having the same average size of adipocytes, the sWAT having the broader cell size distribution will be stiffer. The dispersion of the cell size distribution is present in (A.4) in the form of $C_{v}^{2}$. To assess the experimental values of $C_{v}^{2}$ we will use the data from Table 2 in [2] presented for different values of body mass index. This data was presented as the lipid mass of adipocytes, $M$, which is connected with adipocyte's size, $R$, through relationship $M=\rho_{0} \beta R^{3}$, where $\rho_{0}$ is the density of triglycerides, $\beta$ is the coefficient of cell's topology. To present these experimental data as $C_{v}^{(R)}$ for the cell size, $R$, we apply the method of error propagation, from which

$$
C_{v}^{(M)}=\sqrt{3} C_{v}^{(R)}
$$

Calculated values of $C_{v}^{(R) 2}$ are presented in Table 1 .

As it follows from (A.4), for such low values of $C_{v}^{(R) 2}$ the stiffness of sWAT depends only on the average size of adipocytes $\bar{R}$ and is almost independent of the dispersion of the cell size distribution. This must be a general result showing the robustness of mechanical stability of sWAT to variations of adipocytes' content at least under quasi-physiological conditions.

\subsection{Heterogeneous Cell Population}

Till now we have considered sWAT as the closed-cell foam which consists of homogeneous adipocytes’ population with distributed cell sizes. As shown above, such distribution typically has a minor effect on the mechanical

\begin{tabular}{cccc}
\hline Table 1. Calculated values of & $C_{v}^{(R) 2}$ & for adipocytes from different fat depots (Original data from [2]). \\
\hline Body Mass Index & Abdominal sWAT & Gluteal sWAT & Femoral sWAT \\
\hline$<25$ & 0.08 & 0.03 & 0.05 \\
$25-30$ & 0.05 & 0.03 & 0.05 \\
$>30$ & 0.03 & 0.03 & 0.02 \\
\hline
\end{tabular}


properties of the fat tissue. This situation can be changed if sWAT consists of several different subpopulations of adipocytes having very different sizes and different pericellular structures. This can be, for example, the case in gluteo-femoral and abdominal regions, as the so-called "beige" adipocytes appear. These cells can be produced through transdifferentiation of white adipocytes [19] as well as through production of adipocytes de novo [20], and their appearance can significantly modulate the volume as well as the metabolic activity of sWAT. Production of "beige" cells in humans is connected with activation of the so called UCP1 gene, normally through exposure to cold or through $\beta$-adrenergic stimulation. This activation can take place in a timeframe of hours. Without repeated treatments, this effect is reversible and the "beige" cells characteristically disappear during a timeframe of approximately 4 - 5 weeks [19], thus supposedly being eliminated through a normal sWAT turnover [21].

Through mechanism of transdifferentiation, a heterogeneous and dynamic sWAT structure containing the mixture of clusters of small ("beige") and of big (white) cells in the same sWAT can appear. Taking into account the relatively short time for production and removal of these new small adipocytes, one can conclude that sWAT can be locally subjected to dynamic modulation of its mechanical properties through spatial modification of the cell size distribution.

Thus, the appearance of small adipocytes in sWAT will increase its stiffness (see Appendix A.2). The value of this increment depends on the volume fraction of small adipocytes as well as on the ratio of average adipocytes' sizes in these two populations. For example, if newly produced small adipocytes occupy only $10 \%$ of the fat tissue volume and the average size of big adipocytes is twice that of the average size of the population of small adipocytes, the stiffness of sWAT will be increased by $10 \%$. If however the induced small adipocytes occupy the same volume, but are three times smaller than the original big cells, the stiffness of such sWAT will increase by $20 \%$.

Similar analysis can be done for the yield strength of sWAT, described by the second expression in (3). The following predictions can immediately be made about the influence of sWAT structure modulation onto the yield strength modulus:

- It will decrease by damaging and elimination of adipocytes;

- It will increase by reduction of cell sizes without accompanying cell death;

- It will increase by induction of small adipocytes, which substitute a part of the volume originally occupied by big cells.

From the above, it is clear that potential plastic transformation of sWAT after body contouring, which can at least be partly responsible for non-reversible results, must be strongly dependent on the applied treatment procedure.

\subsection{Cell Shape}

Until now we have considered the adipocytes to be the cells of a regular cubic form. Whereas less symmetric as in the "equiaxed" spherical case, this morphology is still symmetric enough to avoid any stress concentration in some preferential direction. Now we will analyze how the modification of the cell form can influence mechanical properties of sWAT.

Different morphological forms of adipocytes are present in a densely packed sWAT: spherical, cubical, hexagonal, elliptical, etc. Whilst, as discussed above, the main mechanical properties of sWAT are connected with relative density of pericellular structures, the topological peculiarities can significantly influence such characteristics as the yield strength, which is connected to the onset of a plastic behavior. The cell shape anisotropy would be of primary importance here.

In cellular solids, cell shape anisotropy is recognized to be much more important for yielding susceptibility as cell size [22]. Whereas the open-cell foams demonstrate more or less homogeneous volume deformation, the closed-cell structures deform heterogeneously even by relatively low stresses. Under compression this deformation typically propagates in a form of so called deformation bands with main strain almost normally oriented to a band plane. Under tensile deformation, the appearing strain fields generally have more uniform mapping and they normally do not produce any localized deformation bands. Instead of this the local strain peaks can arise at weak locations. For the closed-cell foams described in [22], the strain increment inside the band can exceed the strain outside it by a factor 10 , which is the sign of a cell collapse and material densification. Interestingly, the larger cells are not usually as susceptible to plastic deformation as the smaller cells showing elastic response 
even by high stresses. At the same time, it was shown that the cells inside the deformation band (which were much more susceptible to the permanent deformation as surrounding cells) have whether the non-planar walls with high curvature or special T-shaped intersections between the walls of neighboring cells. The same qualitative results must be also valid for sWAT behavior.

In the same material, the elliptic-shaped cells must be generally more susceptible to plastic deformation as more isotropic “equiaxed” cells. The reason for this is the stress concentration in the places of the maximal curvature of the cell surface. The yielding strength for isotropic cells can be double that of the corresponding elliptical cells which have the same volume. This item was theoretically analyzed in [22]. Therefore, the curvy cells are mechanically "weaker" comparing to isotropic cells and can selectively respond to their deformation with local yielding. Analogously, sWAT morphology with increased number of such “elliptical” adipocytes will have a decreased yielding strength.

Such elliptical morphology of adipocytes can be induced under application of uniaxial tension (in the direction of applied forces) or uniaxial compression (in a perpendicular direction). Elongation of adipocytes of up to $45 \%$ in the direction of applied strain was shown in human sWAT [9]. Elliptic structures can also arise as a result of adipocytes' death and local loss of pericellular structure. As discussed above, such cell loss leads to decrease of both Young's modulus and yields stress in affected tissue.

\section{Possible Applications in Non-Invasive Body Contouring}

Non-invasive and minimal invasive body contouring procedures include applications of various physical factors, which are believed to be able to produce a significant volume modulation of sWAT. Although most of these procedures claim the adipocytes' death to be the main mechanism of this modification, the treatment results are relatively short-term and cannot be temporally compared with those of aesthetic-plastic surgery which are obviously connected with removal of fat cells. As discussed in [1], different physiological processes with various characteristic reaction times can be involved in sWAT modulation. One of important mechanisms of a quick modulation of sWAT is the production of hyaluronan with consequent retention of water in the tissue, which can be non-specifically stimulated by application of different physical forces.

Another possibility for quick sWAT modulation is the short-termed change of mechanical properties of this tissue, e.g. through its strain hardening-the strengthening of a material by plastic deformation. This effect is characterized by the quick increase of material stiffness in a small interval of deformations. An example of such soaring storage modulus describing the stiffness of sWAT under vibratory conditions was given in [23]: sWAT hardening was observed after 250 - 1200 s application of local tissue deformation and it was characterized by almost an order of magnitude increase of the tissue stiffness. This effect is normally reversible and typically disappears after several hours. Micromechanically it must be connected with plastic modification of pericellular collagen structure, which is a similar effect to the solid foams [24].

From above, it is clear that mechanical properties of sWAT are strongly dependent on its structure, which can be from the micromechanical point of view described as the closed-cell foam. Since the stiffness of this structure inversely depends on the average adipocytes' size, the sWAT containing small or big adipocytes must have different mechanical properties. Both elastic and yielding strength moduli must be higher in sWAT containing smaller adipocytes. Consequently, such sWAT must be stiffer and more resistant to the onset of plastic transformation.

From the other side, larger adipocytes can have thicker pericellular structures which would improve the mechanical properties of such hypertrophic tissue. Different treatment methods can modulate these mechanical properties in different ways, sometimes leading to opposite results. For examples, induced death and removal of adipocytes must at least, during the first period after treatment, reduce the stiffness of the fat tissue. This loss can later be compensated through re-arrangement of survived adipocytes and production of new small adipocytes filling the same areas. This phenomenon can be responsible for dynamic modulation of sWAT stiffness which can take several weeks or even months after some non-invasive procedures. Contrary, treatment methods which can cause cell size reduction without cell death (e.g. through induced lipolysis) must demonstrate the increase in sWAT stiffness shortly after intervention.

Different morphologies of adipocytes can also significantly influence the mechanical properties of sWAT. This is especially true in the case of the cells producing wrinkling of their reinforced basement structure or changing their shapes, which produces the surfaces of high curvature. The latter is possible during different 
body contouring procedures connected with the application of tension (pulsed or especially constant "vacuum”) or compression. Under the application of such mechanical forces, the adipocytes change their forms to elliptic, thus producing the regions of high strain concentration which can then additionally be modulated thermo-mechanically. This can consequently lead to local reduction of yielding strength in sWAT, making this tissue plastic and the volume recovery after such procedure not fully reversible.

A special case is presented by inhomogeneous sWAT structure with spatial distribution of clusters of small and big adipocytes. As is seen from (A.8), induction of small adipocytes must increase the stiffness of the fat tissue locally. If the clusters of small adipocytes are distributed in sWAT, the result of their appearance will be the production of inhomogeneous tension field which will be projected onto the skin. Primarily this effect is connected with the appearance of pericellular fibrosis around hypertrophic adipocytes, which was supposed to be an important pathophysiological factor in cellulite [25] and can be considered as its necessary condition. Inhomogeneous cell size distribution of adipocytes can make this effect visible on the skin surface, thus producing the sufficient condition in a pathophysiology of at least mild forms of cellulite. The appearance of small adipocytes in gluteo-femoral area, where cellulite typically appears, can at least partly be explained by the intensive production of small "beige" adipocytes in this area. From this point of view, the treatment strategy in cellulite must include 1) the reduction of pericellular fibrosis, 2) the reduction of cell size dispersion, e.g. through selective elimination of small or big cells from sWAT. This topic will be discussed in more detail elsewhere.

\section{Conclusions}

Micromechanical description of sWAT as a foam structure gives the possibility to receive simple relations for mechanical moduli describing its elastic behavior and plastic susceptibility. Mechanical stiffness of sWAT must be primarily dependent on the average cell size and almost independent of the dispersion of cell sizes in a local adipocytes' population. Whereas the influence of the natural fat renewal process with a rate of $10 \%$ per year must be of minor importance for mechanical properties of sWAT, mass-induced adipocytes' death can substantially reduce local sWAT stiffness. The sWAT containing two or more different subpopulations of adipocytes of very different sizes with a spatially clustered structure can demonstrate significant inhomogeneity of their mechanical properties compared with those of sWAT that consist of a single population of adipocytes. This effect may be an important pathophysiological feature of cellulite. Additionally, modulation of the cell shape can make adipocytes more susceptible to thermo-mechanical stress, thus reducing the strain needed to achieve the plastic deformation in this tissue.

Different non-invasive and minimal invasive body contouring procedures can variably modulate the mechanical properties of sWAT. The methods which can really induce the damage of adipocytes and rupturing of their membrane must demonstrate initial volume loss correlated with reduction of tissue stiffness. The latter can then be improved temporally in the course of replacement of damaged adipocytes by newly generated small cells. Contrary to this, the methods which can locally induce pure lipolysis without correspondent cell death must demonstrate initial increase in sWAT stiffness. Application of mechanical tension or compression in combination with other physical factors can modulate the cell shape thus producing the highly curved surfaces which are more susceptible to appearance of plastic deformation. Application of additional physical factors in such combination must however take into account different orientation of strain fields in sWAT after the application of compressive and tensile deformations.

\section{References}

[1] Kruglikov, I.L. (2014) Processes of Quick and Slow Modulation of Subcutaneous Fat Tissue Volume in Body Contouring Procedures. Journal of Cosmetics, Dermatological Sciences and Applications, 4, 107-116.

[2] Tchoukalova, Y.D., Koutsari, C., Karpyak, M.V., Votruba, S.B., Wendland, E. and Jensen, M.D. (2008) Subcutaneous Adipocytes Size and Body Fat Distribution. American Journal of Clinical Nutrition, 87, 56-63.

[3] Spalding, K.L., Arner, E., Westermark, P.O., Bernard, S., Buchholz, B.A., Bergmann, O., Blomqvist, L., Hoffstedt, J., Näslund, E., Britton, T., Concha, H., Hassan, M., Ryden, M., Frisen, J. and Arner, P. (2008) Dynamics of Fat Cell Turnover in Humans. Nature, 453, 783-787. http://dx.doi.org/10.1038/nature06902

[4] Arner, E., Westermark, P., Spalding, K.L., Britton, T., Ryden, M., Frisen, J., Bernard, S. and Arner, P. (2010) Adipocyte Turnover: Relevance to Human Adipose Tissue Morphology. Diabetes, 59, 105-109. http://dx.doi.org/10.2337/db09-0942 
[5] Monteiro, R., de Castro, P.M.S.T., Calhau, C. and Azevedo, I. (2006) Adipocyte Size and Liability to Cell Death. Obesity Surgery, 16, 804-806. http://dx.doi.org/10.1381/096089206777346600

[6] Fournier, L. and Joos, B. (2003) A Lattice Model for the Kinetics of Rupture of Fluid Bilayer Membranes. Physical Review E, 67, Article ID: 051908. http://dx.doi.org/10.1103/PhysRevE.67.051908

[7] Comley, K. and Fleck, N.A. (2010) A Micromechanical Model for the Young’s Modulus of Adipose Tissue. International Journal of Solids and Structures, 47, 2982-2990. http://dx.doi.org/10.1016/j.ijsolstr.2010.07.001

[8] Comley, K. and Fleck, N.A. (2010) The Toughness of Adipose Tissue: Measurements and Physical Basis. Journal of Biomechanics, 43, 1823-1826. http://dx.doi.org/10.1016/j.jbiomech.2010.02.029

[9] Alkhouli, N., Mansfeld, J., Green, E., Bell, J., Knight, B., Liversedge, N., Tham, J. C., Welbourn, R., Shore, A.C., Kos, K. and Winlove, C.P. (2013) The Mechanical Properties of Human Adipose Tissue and Their Relatioships to the Structure and Composition of the Extracellular Matrix. American Journal of Physiology. Endocrinology and Metabolism, 305, E1427-E1435. http://dx.doi.org/10.1152/ajpendo.00111.2013

[10] Chun, T.-H., Hotary, K.B., Sabeh, F., Saltiel, A.R., Allen, E.D. and Weiss, S.J. (2006) A Pericellular Collagenase Directs the 3-Dimensional Development of White Adipose Tissue. Cell, 125, 577-591. http://dx.doi.org/10.1016/j.cell.2006.02.050

[11] Khan, T., Muise, E.S., Iyengar, P., Wang, Z.V., Chandalia, M., Abate, N., Zhang, B.B., Bonaldo, P., Chua, S. and Scherer, P.E. (2009) Metabolic Dysregulation and Adipose Tissue Fibrosis: Role of Collagen VI. Molecular and Cellular Biology, 29, 1575-1591. http://dx.doi.org/10.1128/MCB.01300-08

[12] Divoux, A., Tordjman, J., Lacasa, D., Veyrie, N., Hugol, D., Aissat, A., Basdevant, A., Guerre-Millo, M., Poitou, C., Zucker, J.-D., Bedossa, P. and Clement, K. (2010) Fibrosis in Human Adipose Tissue: Composition, Distribution, and Link with Lipid Metabolism and Fat Mass Loss. Diabetes, 59, 2817-2825. http://dx.doi.org/10.2337/db10-0585

[13] Alexopoulos, L.G., Youn, I., Bonaldo, P. and Guilak, F. (2009) Developmental and Osteoarthritic Changes in Col6a1Knockout Mice: Biomechanics of Type VI Collagen in the Cartilage Pericellular Matrix. Arthritis \& Rheumatism, 60, 771-779. http://dx.doi.org/10.1002/art.24293

[14] Gibson, L.J. and Ashby, M.F. (1997) Cellular Solids: Structure and Properties. 2nd Edition, Cambridge University Press, Cambridge.

[15] Cinti, S., Mitchell, G., Barbatelli, G., Murano, I., Ceresi, E., Faloia, E., Wang, S., Fortier, M., Greenberg, A.S. and Obin, M.S. (2005) Adipocytedeath Defines Macrophage Localization and Function in Adipose Tissue of Obese Mice and Humans. Journal of Lipid Research, 46, 2347-2355. http://dx.doi.org/10.1194/jlr.M500294-JLR200

[16] Strissel, K.J., Stancheva, Z., Miyoshi, H., Perfeld II, J.W., DeFuria, J., Jick, Z., Greenberg, A.S. and Obin, M.S. (2007) Adipocyte Death, Adipose Tissue Remodeling, and Obesity Complications. Diabetes, 56, 2910-2918. http://dx.doi.org/10.2337/db07-0767

[17] Kang, L., Lantier, L., Kennedy, A.J., Bonner, J.S., Mayes, W.H., Bracy, D.P., Bookbinder, L.H., Hasty, A.H., Thompson, C.B. and Wasserman, D.H. (2013) Hyaluronan Accumulates with High Fat Feeding and Contributes to Insulin Resistance. Diabetes, 62, 1888-1896. http://dx.doi.org/10.2337/db12-1502

[18] Kruglikov, I.L. (2013) Is the Depletion of Hyaluronan in Hypertrophic Fat Tissue a Key Event in Body-Contouring Procedures? The American Journal of Cosmetic Surgery, 30, 244-245.

[19] Rosenwald, M., Perdikari, A., Rülicke, T. and Wolfrum, C. (2013) Bi-Directional Interconversion of Brite and White Adipocytes. Nature Cell Biology, 15, 659-667. http://dx.doi.org/10.1038/ncb2740

[20] Wang, Q.A., Tao, C., Gupta, R.K. and Scherer, P.E. (2013) Tracking Adipogenesis during White Adipose Tissue Development, Expansion and Regeneration. Nature Cell Biology, 19, 1338-1344. http://dx.doi.org/10.1038/nm.3324

[21] Harms, M. and Seale, P. (2013) Brown and Beige Fat: Development, Function and Therapeutic Potential. Nature Medicine, 19, 1252-1263. http://dx.doi.org/10.1038/nm.3361

[22] Bart-Smith, H., Bastawros, A.-F., Mumm, D.R., Evans, A.G., Sypeck, D.J. and Wadley, H.N.G. (1998) Compressive Deformation and Yielding Mechanisms in Cellular Al Alloys Determined Using X-Ray Tomography and Surface Strain Mapping. Acta Materialia, 46, 3583-3592. http://dx.doi.org/10.1016/S1359-6454(98)00025-1

[23] Geerligs, M., Peters, G.W.M., Ackermans, P.A.J., Oomens, C.W.J. and Baaijens, F.P.T. (2010) Does Subcutaneous Adipose Tissue Behave as an (Anti-)thixotropic Material?” Journal of Biomechanics, 43, 1153-1159. http://dx.doi.org/10.1016/j.jbiomech.2009.11.037

[24] Mangipudi, K.R., van Buuren, S.W. and Onck, P.R. (2010) The Microstructural Origin of Strain Hardening in TwoDimensional Open-Cell Metal Foams. International Journal of Solids and Structures, 47, 2081-2096. http://dx.doi.org/10.1016/j.ijsolstr.2010.04.009

[25] Kruglikov, I.L. (2012) The Pathophysiology of Cellulite: Can the Puzzle Eventually Be Solved? Journal of Cosmetics, Dermatological Sciences and Applications, 2, 1-7. http://dx.doi.org/10.4236/jcdsa.2012.21001 


\section{Appendix}

\section{A1. Effect of Adipocytes' Size Distribution}

To describe the influence of the cell size distribution on the mechanical properties of the tissue, let us assess the Young's modulus of compound structure containing the mixture of cells with some cell size distribution, $\varphi(R, h)$, which can generally be a function of both variables - cell size, $R$, and thickness of the cell's pericellular structure, $h$. The upper limit of $\tilde{E}$ can be then assesses from the rule of mixtures as

$$
\tilde{E}=E_{s} \iint \frac{h}{R} \varphi(R, h) \mathrm{d} R \mathrm{~d} h
$$

Let us consider the following model of sWAT as the closed-cell foam:

- the thickness of pericellular structure is independent of the cell size, i.e. $h=$ const;

- $\varphi(R)$ is described by a gamma-distribution, $\varphi(R)=\frac{\lambda^{\alpha} R^{\alpha-1}}{\Gamma(\alpha)} \mathrm{e}^{-\lambda R}$, which must be a good approximation to make the estimations.

In this case, one can easily receive the upper limit approximation which corresponds to the Voigt model for composite materials:

$$
\max \tilde{E}=E_{s} h \int_{0}^{\infty} \frac{\lambda^{\alpha} R^{\alpha-1}}{R \Gamma(\alpha)} \mathrm{e}^{-\lambda R} \mathrm{~d} R=E_{s} \frac{h}{\bar{R}} \frac{1}{1-C_{v}^{2}}
$$

Here $\bar{R}$ is the average adipocytes' size and $C_{v}$ is the coefficient of variation of these sizes. The lower limit of $\tilde{E}$ corresponding to the Reuss model for composite materials can be also easily calculated as

$$
\min \tilde{E}=E_{s} h \int_{0}^{\infty} R \frac{\lambda^{\alpha} R^{\alpha-1}}{\Gamma(\alpha)} \mathrm{e}^{-\lambda R} \mathrm{~d} R=E_{\mathrm{s}} \frac{h}{\bar{R}}
$$

Thus the effective Young's modulus of sWAT belongs to the interval

$$
E_{s} \frac{h}{\bar{R}} \leq \tilde{E} \leq E_{s} \frac{h}{\bar{R}} \frac{1}{1-C_{v}^{2}}
$$

\section{A2. Heterogeneous Cell Population}

To make a qualitative analysis, let us consider a simple case of this distribution with two types of cells: the first type is the small cells distributed around the average size $\overline{R_{1}}$, the second type is the big cells distributed around the size $\overline{R_{2}}$. If these distributions don't overlap, it is enough to consider the first approximation of the cells, of both populations, to be uniform in size, i.e. having the distributions $\delta\left(R-\overline{R_{1}}\right)$ and $\delta\left(R-\overline{R_{2}}\right)$, respectively.

Applying the rule of mixtures, the upper limit for effective Young's modulus of such mixed tissue can be presented in the form

$$
\max \tilde{E} \leq \mu \widetilde{E_{1}}+(1-\mu) \widetilde{E_{2}}
$$

here $\widetilde{E_{1}}$ and $\widetilde{E_{2}}$ are the effective Young's moduli of the pericellular structure of the cells types 1 and 2, respectively; $\mu$ is a volume fraction of the cells type 1. Taking relationship (3) into account, we can rewrite (A.5) in the form

$$
\max \tilde{E} \leq E_{s}\left\{\mu \frac{h_{1}}{\overline{R_{1}}}+(1-\mu) \frac{h_{2}}{\overline{R_{2}}}\right\}
$$

Let us compare this value with the Young's modulus of a fat tissue containing only one type of cells with the size $\overline{R_{2}}$, which is $\widetilde{E_{0}} \leq E_{s} \frac{h_{2}}{\overline{R_{2}}}$ :

$$
\frac{\tilde{E}-\widetilde{E_{0}}}{\widetilde{E_{0}}}=\mu\left(\frac{h_{1}}{h_{2}} \frac{\overline{R_{2}}}{\overline{R_{1}}}-1\right)
$$


Now we can consider the following special cases.

a) Small cells have no or very weak reinforced basement structure, i.e. $h_{1} \ll h_{2}$.

In this case $\tilde{E}<\widetilde{E_{0}}$ and the small adipocytes appear micromechanically case as "holes". This is qualitatively the same result as for dead cells described above in 3.1.

b) Small cells have the same pericellular structure as the big cells, i.e. $h_{1}=h_{2}=h$. For this,

$$
\frac{\tilde{E}-\widetilde{E_{0}}}{\widetilde{E_{0}}}=\mu\left(\frac{\overline{R_{2}}}{\overline{R_{1}}}-1\right)>0
$$

Thus $\tilde{E}>\widetilde{E_{0}}$ and the small adipocytes appear micromechanically in this case as "rigid" inclusions. 